\title{
Hypoxic Epithelial Necrosis Triggers Neutrophilic Inflammation via IL-1 Receptor Signaling in Cystic Fibrosis Lung Disease
}

\begin{abstract}
Benedikt Fritzsching ${ }^{1,2 *}$, Zhe Zhou-Suckow ${ }^{1,2 \star}$, Joanna B. Trojanek ${ }^{1,2}$, Susanne C. Schubert ${ }^{1,2}$, Jolanthe Schatterny ${ }^{1,2}$, Stephanie Hirtz ${ }^{1,2}$, Raman Agrawal ${ }^{1,2}$, Thomas Muley ${ }^{2,3}$, Nicolas Kahn ${ }^{2,4}$, Carsten Sticht ${ }^{5}$, Nikolas Gunkel ${ }^{6}$, Tobias Welte $^{7,8}$, Scott H. Randell ${ }^{9}$, Florian Länger ${ }^{8,10}$, Philipp Schnabel ${ }^{2,11}$, Felix J. F. Herth ${ }^{2,4}$, and Marcus A. Mall ${ }^{1,2}$

${ }^{1}$ Department of Translational Pulmonology and ${ }^{11}$ Institute of Pathology, University of Heidelberg, Heidelberg, Germany; ${ }^{2}$ Translational Lung Research Center Heidelberg, Member of the German Center for Lung Research, Heidelberg, Germany; ${ }^{3}$ Translational Research Unit and ${ }^{4}$ Department of Pneumology and Critical Care Medicine, Thoraxklinik at the University Hospital Heidelberg, Heidelberg,

Germany; ${ }^{5}$ Medical Research Center, Medical Faculty Mannheim, University of Heidelberg, Mannheim, Germany; ${ }^{6}$ Cancer Drug Development, German Cancer Research Center, Heidelberg, Germany; ${ }^{7}$ Department of Respiratory Medicine and ${ }^{10}$ Institute of Pathology, Hannover Medical School, Hannover, Germany; ${ }^{8}$ Biomedical Research in Endstage and Obstructive Lung Disease Hannover, Member of the German Center for Lung Research, Hannover, Germany; and ${ }^{9}$ Cystic Fibrosis Center and Department of Cell Biology and Physiology, The University of North Carolina at Chapel Hill, Chapel Hill, North Carolina
\end{abstract}

\section{Abstract}

Rationale: In many organs, hypoxic cell death triggers sterile neutrophilic inflammation via IL-1R signaling. Although hypoxia is common in airways from patients with cystic fibrosis (CF), its role in neutrophilic inflammation remains unknown. We recently demonstrated that hypoxic epithelial necrosis caused by airway mucus obstruction precedes neutrophilic inflammation in Scnn1b-transgenic (Scnn1b-Tg) mice with CF-like lung disease.

Objectives: To determine the role of epithelial necrosis and IL-1R signaling in the development of neutrophilic airway inflammation, mucus obstruction, and structural lung damage in CF lung disease.

Methods: We used genetic deletion and pharmacologic inhibition of IL-1R in Scnn 1b-Tg mice and determined effects on airway epithelial necrosis; levels of IL-1 $\alpha$, keratinocyte chemoattractant, and neutrophils in bronchoalveolar lavage; and mortality, mucus obstruction, and structural lung damage. Furthermore, we analyzed lung tissues from
21 patients with $\mathrm{CF}$ and chronic obstructive pulmonary disease and 19 control subjects for the presence of epithelial necrosis.

Measurements and Main Results: Lack of IL-1R had no effect on epithelial necrosis and elevated IL- $1 \alpha$, but abrogated airway neutrophilia and reduced mortality, mucus obstruction, and emphysema in Scnn1b-Tg mice. Treatment of adult Scnn1b-Tg mice with the IL-1R antagonist anakinra had protective effects on neutrophilic inflammation and emphysema. Numbers of necrotic airway epithelial cells were elevated and correlated with mucus obstruction in patients with $\mathrm{CF}$ and chronic obstructive pulmonary disease.

Conclusions: Our results support an important role of hypoxic epithelial necrosis in the pathogenesis of neutrophilic inflammation independent of bacterial infection and suggest IL-1R as a novel target for antiinflammatory therapy in CF and potentially other mucoobstructive airway diseases.

Keywords: airway inflammation; airway epithelium; mucus obstruction; cystic fibrosis; chronic obstructive pulmonary disease
Recent studies in infants with cystic fibrosis (CF) diagnosed by newborn screening identified neutrophilic inflammation and air trapping caused by small airways mucus obstruction as early abnormalities that are present in most infants often in the absence of detectable bacterial infection and respiratory symptoms $(1,2)$. Neutrophilic inflammation has been implicated in

(Received in original form September 5, 2014; accepted in final form January 13, 2015)

*These authors contributed equally to this work.

Supported by grants from the Deutsche Forschungsgemeinschaft (MA 2081/3-2 and MA 2081/4-1), National Institutes of Health (DK065988), and the European Commission (Seventh Framework Program Project No. 603038 CFMatters).

Author Contributions: Conception and design of the study, B.F., Z.Z.-S., J.B.T., S.C.S., R.A., T.M., N.G., P.S., and M.A.M. Acquisition, analysis, and

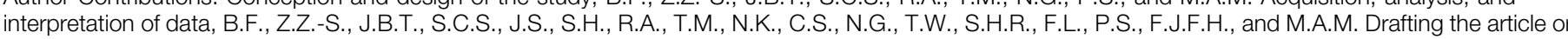

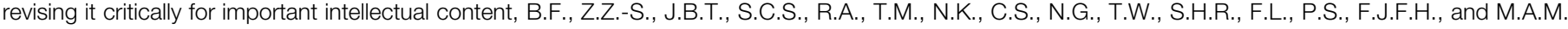

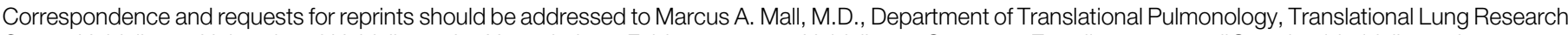
Center Heidelberg, University of Heidelberg, Im Neuenheimer Feld 350, 69120 Heidelberg, Germany. E-mail: marcus.mall@med.uni-heidelberg.de

This article has an online supplement, which is accessible from this issue's table of contents at www.atsjournals.org

Am J Respir Crit Care Med Vol 191, Iss 8, pp 902-913, Apr 15, 2015

Copyright (C) 2015 by the American Thoracic Society

Originally Published in Press as DOI: 10.1164/rccm.201409-16100C on January 21, 2015

Internet address: www.atsjournals.org 


\section{At a Glance Commentary}

\section{Scientific Knowledge on the}

Subject: Recent studies in infants with cystic fibrosis (CF) detected neutrophilic inflammation and small airways mucus obstruction as early abnormalities that are often present in the apparent absence of bacterial infection. IL-1 $\alpha$ derived from necrotic cells has been identified as a potent trigger of sterile neutrophilic inflammation. Although mucusobstructed CF airways exhibit areas of hypoxia (i.e., a common cause of necrotic cell death), the role of hypoxic epithelial necrosis, IL- $1 \alpha$, and IL-1R signaling in $\mathrm{CF}$ airway inflammation remains unknown.

\section{What This Study Adds to the}

Field: This study identifies hypoxic epithelial necrosis with release of IL- $1 \alpha$ and activation of IL-1R-myeloid differentiation primary response gene 88 signaling as a potent trigger of neutrophilic airway inflammation in mice with CF-like lung disease. Furthermore, we demonstrate an increased abundance of necrotic epithelial cells in mucus-obstructed airways from patients with CF and chronic obstructive pulmonary disease. These results support an important role of hypoxic epithelial necrosis in the in vivo pathogenesis of neutrophilic inflammation independent of bacterial infection and suggest IL-1R as a novel target for antiinflammatory therapy in patients with $\mathrm{CF}$ and potentially other mucoobstructive lung diseases.

several disease processes in CF lung disease including dysregulated epithelial ion transport and structural lung damage, and neutrophil elastase (NE), a major neutrophil product, was identified as a key risk factor for bronchiectasis and lung function decline in children with CF (3-9). Furthermore, evidence suggests that mediators released in neutrophilic airway inflammation contribute to goblet cell metaplasia and mucus hypersecretion under certain, but not all pathophysiologic conditions (9-11). However, the in vivo pathogenesis of neutrophilic inflammation in the absence of apparent bacterial infection in early CF lung disease remains unknown.

Besides bacterial infection, cell injury and necrosis constitutes another principal stimulus of inflammation. Evidence from common diseases caused by tissue hypoxia, such as myocardial infarction and ischemic stroke, demonstrated that hypoxic cell death provides a potent trigger of sterile neutrophilic inflammation $(12,13)$. Whereas inflammation in infection is stimulated by binding of microbial components to diverse Toll-like receptors (TLR) that signal through the common adaptor protein myeloid differentiation primary response gene 88 (MyD88) (14), recent studies in mouse models of sterile peritonitis and liver necrosis identified activation of IL-1R as a key pathway linking necrosis with inflammation in the absence of infection $(12,15)$. IL-1R is a cytokine receptor that also uses MyD88 signaling and is activated by IL- $1 \alpha$ induced by dying cells. Although reduced oxygen tension caused by airway mucus plugging and increased epithelial oxygen consumption caused by increased absorption of airways surface liquid is a characteristic abnormality of CF airways $(16,17)$, the role of hypoxic necrosis and IL-1R-MyD88 signaling in CF airways inflammation has not been studied.

In previous studies using Scnn $1 b-\mathrm{Tg}$ mice as a model of CF lung disease $(18,19)$, we demonstrated that airway surface dehydration, a key disease mechanism in $\mathrm{CF}$, causes airway mucus obstruction in the first days of life, that early mucus plugging is associated with systemic and cellular hypoxia of airway epithelia, and that a subset of Clara cell undergoes hydropic degeneration and necrosis (20).

Furthermore, we observed that the appearance of necrotic cells preceded the onset of airway neutrophilia in neonatal Scnn $1 b$-Tg mice (20). The abundance of necrotic epithelial cells correlated with the severity of airway mucus obstruction (21) and was decreased when mucus plugging was reduced by preventive amiloride treatment in neonatal Scnn1b-Tg mice (22).

The aim of this study was, therefore, to determine the role of airway epithelial necrosis and IL-1R-MyD88 pathway activation in the in vivo pathogenesis of neutrophilic inflammation associated with mucus obstruction. To achieve this goal, we crossed Scnn1b-Tg mice with
IL-1R-deficient mice (Il1r1 $\left.1^{-/-}\right)$and determined effects of genetic deletion of Ill $r$ on airway epithelial necrosis, IL- $1 \alpha$ levels, airway neutrophilia, mucus obstruction, structural lung damage, and mortality. Furthermore, we used the IL-1R antagonist (IL-1Ra) anakinra (15) to determine effects of pharmacologic inhibition of IL-1R signaling in adult Scnn $1 b$-Tg mice with established lung disease. In addition, we compared expression profiles of genes implicated in airway inflammation in lungs from neonatal Scnn $1 b$-Tg mice with mild versus severe mucus plugging using wholegenome microarray analysis. Finally, we used in situ hybridization and immunohistochemistry to localize IL- $1 \alpha$ expression in mouse lungs, and evaluated airways sections from patients with $\mathrm{CF}$ and chronic obstructive pulmonary disease (COPD) for the presence of necrotic epithelial cells. Some of the results of these studies have been previously reported in the form of an abstract (23).

\section{Methods}

\section{Human Lung Tissues}

Lung tissues from 13 patients with CF $(29.2 \pm 1.9 \mathrm{yr})$ who underwent lung transplantation and eight patients with COPD (66.6 $\pm 3.3 \mathrm{yr}$ ) diagnosed according to Global Initiative for Chronic Obstructive Lung Disease guidelines (24) were included in this study. Lung tissues from 16 never smokers $(68.6 \pm 2.4 \mathrm{yr})$ with normal lung function who underwent surgical resection for suspected lung cancer and from three donors with no lung disease $(15.3 \pm 5.5 \mathrm{yr})$ were included as control subjects. This study was approved by the ethics committees of the University of Heidelberg and the Hannover Medical School, and the institutional review board of the University of North Carolina at Chapel Hill. Informed written consent was obtained from all subjects. Additional information is provided in the online supplement.

\section{Experimental Animals}

All animal studies were approved by the Regierungspräsidium Karlsruhe, Germany. Scnn1b-Tg mice (18) were backcrossed onto the C57BL/6 background (21) and intercrossed with $I l 1 r 1^{-1-}$ mice on the C57BL/6 background (Jackson Laboratory, Bar Harbor, ME) (25) to 
generate Scnn $1 b$-Tg/Illr1 $1^{-/-}$, Scnn1b-Tg, $\operatorname{Illr} 1^{-/-}$, and wild-type (WT) mice in the second filial generation. Offspring were genotyped as described $(18,25)$ and mice were studied at neonatal $(5 \mathrm{~d})$ or adult (6 wk) ages. Microarray analyses were performed in neonatal ( $3 \mathrm{~d}$ old) and anakinra treatment studies in adult (4-6 wk old) Scnn1b-Tg mice and WT littermate control animals on the original mixed genetic background (C57BL/6N $\times \mathrm{C} 3 \mathrm{H} /$ $\mathrm{HeN})(18,20)$. Mice were housed in a specific pathogen-free animal facility and had free access to chow and water.

\section{Anakinra Treatment}

Adult (4-6 wk old) Scnn1b-Tg mice and WT littermates were treated with the IL$1 \mathrm{Ra}$ anakinra (Kineret; Swedish Orphan Biovitrum AB, Stockholm, Sweden) (15) by subcutaneous injection of $200 \mathrm{mg} / \mathrm{kg}$ body weight, or equal volumes of vehicle $(\mathrm{NaCl}$ $0.9 \%$ ) alone, twice per day for a period of 7 days. Twelve hours after the last treatment, bronchoalveolar lavage (BAL) was performed and lungs were removed for further analysis as described in the online supplement.

\section{BAL Cell Counts and Cytokine Measurements}

Mice were anesthetized via intraperitoneal injection of $120 \mathrm{mg} / \mathrm{kg}$ ketamine and $16 \mathrm{mg} / \mathrm{kg}$ xylazine. BAL, differential cell counts, and ELISA of cytokines were performed as previously described (20). Additional details are provided in the online supplement.

\section{Histology and Morphometry}

Degenerative airway epithelial cells were identified by morphologic criteria (i.e., cell swelling with cytoplasmic vacuolization) and quantified by counting the number of degenerative cells per millimeter of the basement membrane. Histologic and morphometric analyses of airway mucus content and assessment of distal airspace enlargement and alveolar wall destruction were performed as previously described $(20,26,27)$ and detailed in the online supplement.

\section{Immunohistochemistry}

Immunohistochemical staining for IL- $1 \alpha$ was performed using a goat polyclonal antimouse-IL- $1 \alpha$ antibody (R\&D Systems, Minneapolis, $\mathrm{MN}$ ) as previously described (28) and detailed in the online supplement.

\section{RNA In Situ Hybridization}

Nonradioactive in situ hybridization of IL- $1 \alpha$ transcripts was performed on $10-\mu \mathrm{m}$ sections of paraffin-embedded lung tissues using custom designed $5^{\prime}$ and $3^{\prime}$ DIG-labeled IL- $1 \alpha$ antisense and sense locked nucleic acid-modified oligonucleotide probes (Exiqon, Woburn, MA) as previously described (29) and detailed in the online supplement.

\section{Microarray Analysis}

Microarray analysis was performed using Mouse Genome 430 2.0 Microarrays (Affymetrix, Santa Clara, CA) and quantitative real-time reverse transcriptase polymerase chain reaction was performed for confirmation of candidate genes as described in the online supplement.

\section{Statistics}

Data were analyzed with SigmaStat version 3.1 (Systat Software, Erkrath, Germany) and are reported as mean \pm SEM. Statistical analyses were performed using Student $t$ test, Mann-Whitney rank sum test, oneway analysis of variance, chi-square test, and Pearson product-moment correlation as appropriate and $P$ less than 0.05 was accepted to indicate statistical significance.

\section{Results}

\section{Lack of IL-1R Reduces Neutrophilic Airway Inflammation Associated with Epithelial Necrosis in Scnn1b-Tg Mice} To determine if IL-1R is implicated in the onset of neutrophilic airway inflammation associated with airway epithelial necrosis in early lung disease in Scnn1b-Tg mice (20), we compared numeric densities of degenerative airway epithelial cells, BAL inflammatory cell counts, macrophage size, and levels of the keratinocyte chemoattractant (KC), IL-1 $\alpha$, IL-1 $\beta$, and IL-1Ra in 5-day-old neonatal Scnn $1 b-\mathrm{Tg} /$ Illr1 $1^{-/-}$mice with Scnn $1 b$-Tg, Illr1 $1^{-/-}$and WT littermates (Figure 1). Degenerative airway epithelial cells were rarely observed in WT and Illr1 ${ }^{-/-}$ mice, but were significantly increased in Scnn1b-Tg mice (Figures 1A and 1B). Epithelial necrosis in neonatal Scnn $1 b-\mathrm{Tg}$ mice was associated with an increase in macrophages and neutrophils, increased macrophage size (Figure 1C), and elevated levels of KC, IL-1 $\alpha$, IL-1 $1 \beta$, and IL-1Ra in BAL (Figure 1D). Lack of IL-1R had no effect on increased levels of degenerative epithelial cells, BAL macrophages, and IL- $1 \alpha$, but airway neutrophilia was abrogated and $\mathrm{KC}$, IL-1 $\beta$, and IL-1Ra were significantly reduced in Scnn1b-Tg/Il1r1 $1^{-/-}$mice compared with Scnn $1 b$-Tg littermates.

To localize IL- $1 \alpha$ mRNA and protein expression in lungs of neonatal WT and Scnn1b-Tg mice, we performed in situ hybridization and immunohistochemistry (Figure 2). With both techniques, we obtained distinct IL- $1 \alpha$ signals in the epithelium lining the conducting airways and to a lesser extent in lung parenchyma of WT and Scnn $1 b$-Tg mice (Figure 2). Consistent with the translocation of IL- $1 \alpha$ protein to the nucleus (13), immunoreactive signals were clearly detected in the cytoplasm and nucleus of epithelial cells, whereas only faint staining was observed in macrophages sporadically detected in lung sections from WT and Scnn1b-Tg mice (Figure 2B; see Figure E1 in the online supplement). Taken together, these results show constitutive expression of IL- $1 \alpha$ by the airway epithelium and lung parenchyma, and suggest that epithelial cells undergoing necrosis constitute a major source of increased IL- $1 \alpha$ levels in neonatal Scnn $1 b$-Tg mice.

In 6-week-old adult Scnn1b-Tg mice, absolute numbers of degenerative airway epithelial cells were lower than at neonatal ages, but remained elevated compared with WT control animals (Figure 3A). Similar to the results obtained in the neonatal studies, lack of IL-1R had no effects on the level of degenerative airway cells (Figure 3A), but neutrophils and $\mathrm{KC}$ were significantly reduced in BAL from Scnn $1 b$-Tg/Il1r $1^{-/-}$mice compared with Scnn 1b-Tg mice (Figures 3B and 3C). Collectively, these results demonstrate that IL-1R signaling plays an important role in the initiation and perpetuation of airway neutrophilia and suggest that necrotic cell death and release of IL- $1 \alpha$ in mucusobstructed hypoxic airways contribute to IL-1R-dependent neutrophilic airway inflammation in Scnn1b-Tg mice.

\section{Lack of IL-1R Reduces Airway Mucus Obstruction and Mortality in Scnn1b-Tg Mice}

Because chronic neutrophilic inflammation is associated with airway mucus plugging, we determined effects of genetic deletion of IL-1R on mucus obstruction and related mortality in Scnn1b-Tg mice (20). Consistent with spontaneous postnatal 
A

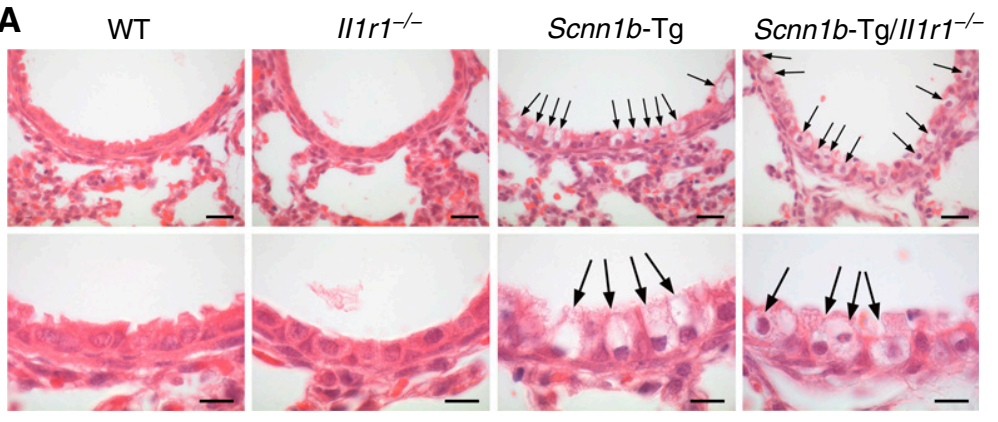

C

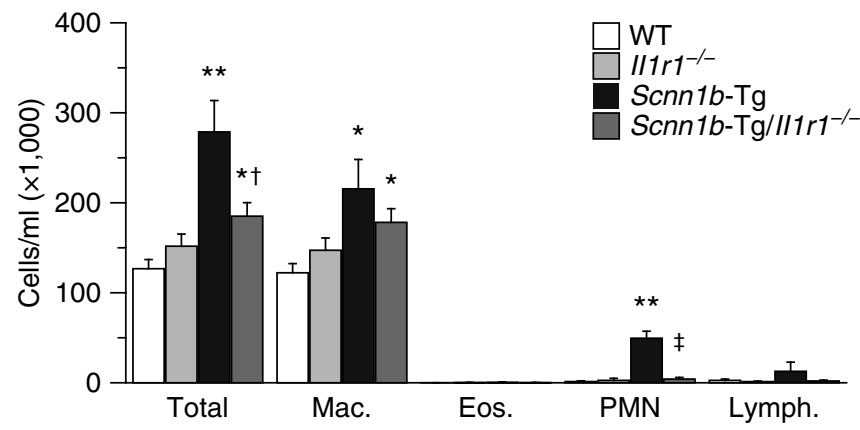

B
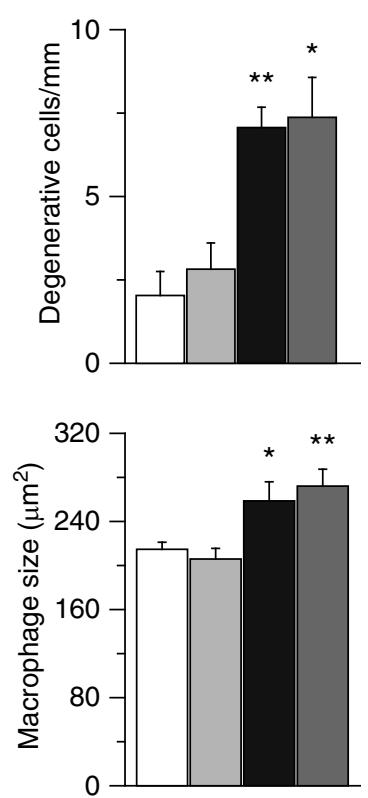
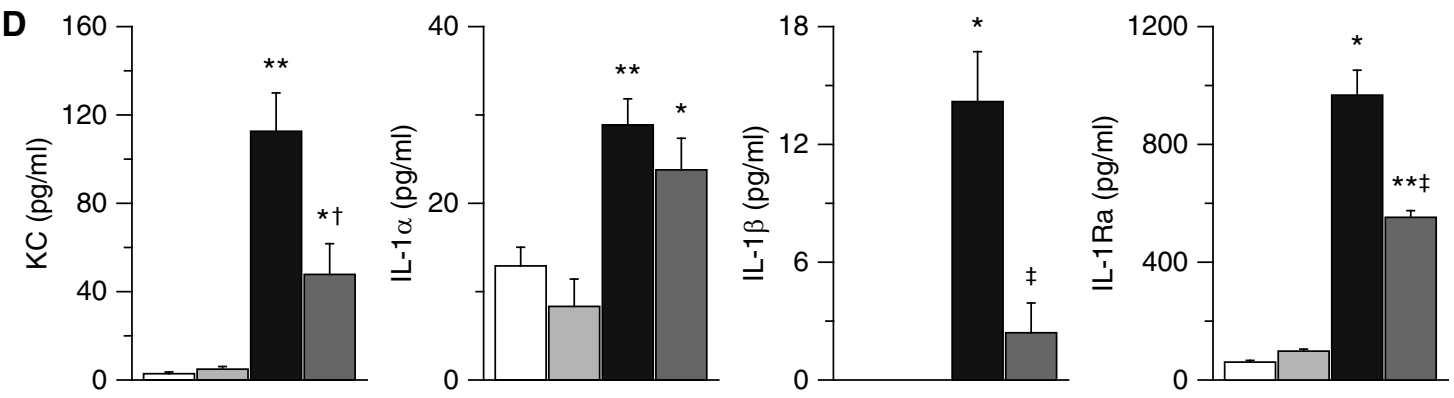

Figure 1. Lack of IL-1R reduces neutrophilic airway inflammation associated with epithelial necrosis in neonatal Scnn $1 b$-Tg mice. Comparison of airway epithelial necrosis $(A$ and $B$ ), bronchoalveolar lavage (BAL) inflammatory cell counts and macrophage size $(C)$ and keratinocyte chemoattractant (KC), IL$1 \alpha, I L-1 \beta$, and IL-1Ra concentrations (D) in 5-day-old wild-type (WT), $/ 11 r 1^{-/-}$, Scnn1b-Tg, and Scnn $1 b$-Tg/ IIr $11^{-1-}$ mice. (A) Representative airway sections were stained with hematoxylin and eosin to determine the number of degenerative epithelial cells (arrows). Scale bars = $20 \mu \mathrm{m}$ (top) and $10 \mu \mathrm{m}$ (bottom). (B) Summary of degenerative epithelial cells per millimeter of the basement membrane. $n=5-10$ mice per group from 16 litters. ${ }^{*} P<0.01$ and ${ }^{\star \star} P \leqslant 0.001$ compared with WT mice. (C) BAL cell counts and macrophage size. $n=8-18$ mice per group from 22 litters. ${ }^{\star} P<0.01$ and ${ }^{\star \star} P \leqslant 0.001$ compared with WT mice. ${ }^{\dagger} P<0.05$ and ${ }^{\ddagger} P \leqslant 0.001$ compared with Scnn $1 b-T g$ mice. (D) KC, IL-1 $\alpha, I L-1 \beta$, and IL-1Ra concentration in BAL. $\mathrm{n}=4-10$ mice per group from $15-20$ litters. ${ }^{\star} P<0.05$ and ${ }^{\star \star} P \leqslant 0.001$ compared with WT mice. ${ }^{\dagger} P<0.05$ and ${ }^{\ddagger} P<0.01$ compared with $S c n n 1 b-T g$ mice. Eos. $=$ eosinophils; Lymph. = lymphocytes; Mac. = macrophages; $\mathrm{PMN}=$ neutrophils.

mortality observed in previous studies (18, 20 ), the percentage of surviving Scnn $1 b$-Tg mice was significantly lower than the expected mendelian ratio for the intercross of Scnn $1 b$-Tg with $I l 1 r 1^{-/-}$mice, whereas Scnn $1 b-\mathrm{Tg} /$ Ill $r 1^{-/-}$mice were protected from postnatal mortality (Figure 4A). Morphometric analysis of airway sections from 6-week-old adult mice demonstrated that airway mucus obstruction in Scnn1b$\mathrm{Tg}$ mice was significantly reduced in Scnn $1 b-\mathrm{Tg} /$ Illr1 $1^{-/-}$mice (Figures $4 \mathrm{~B}$ and $4 \mathrm{C})$. The notion that IL-1R signaling is implicated in airway mucus obstruction was supported by independent studies using whole-genome cDNA microarray analysis of lungs from neonatal Scnn $1 b$-Tg mice on a mixed genetic background $(\mathrm{C} 57 \mathrm{BL} / 6 \mathrm{~N} \times \mathrm{C} 3 \mathrm{H} / \mathrm{HeN})$ that develop more heterogeneous disease $(18,20)$ and were selected for exhibiting either mild ( $\leqslant 20 \%$ of luminal area) or severe $(\geqslant 80 \%$ of luminal area) tracheal mucus obstruction (Figures 5A and 5B).

Seeking for genes that were differentially expressed in Scnn1b-Tg mice with mild versus severe mucus plugging, with a focus on genes implicated in inflammation using custom gene set and gene set enrichment analysis, we found that Il1ra was differentially up-regulated in Scnn1b-Tg mice with mild mucus obstruction (Figure 5D). Significant up-regulation of Illra (approximately threefold) in lungs from Scnn1b-Tg mice with mild versus severe airway obstruction was confirmed by real-time reverse transcriptase polymerase chain reaction (Figure 5E). Of note, the number of necrotic airway cells did not differ in Scnn1b-Tg mice with mild versus severe tracheal obstruction indicating that differences in Illra expression, rather than 


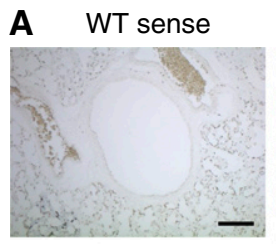

WT antisense
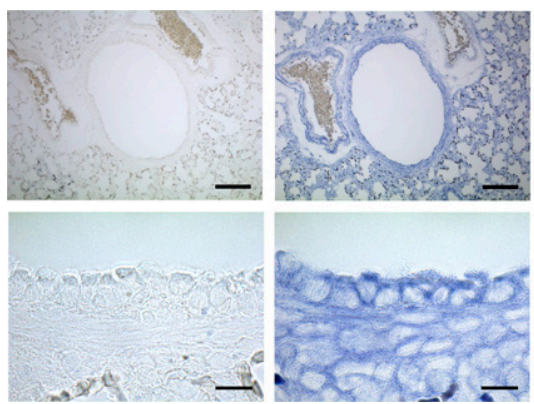

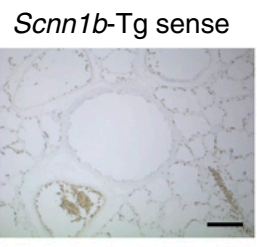

Scnn $1 b$-Tg antisense

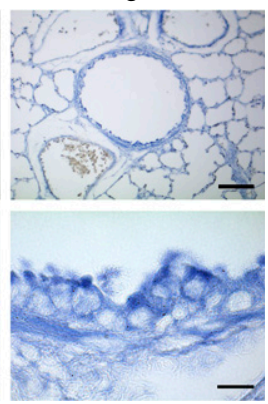

B WT control

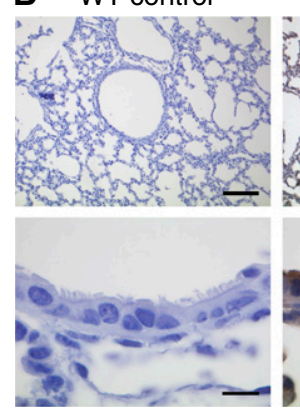

WT IL-1 $\alpha$

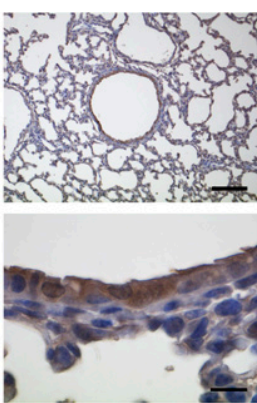

Scnn $1 b$-Tg control

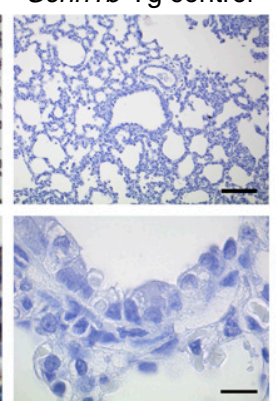

Scnn $1 b-\operatorname{Tg} \mathrm{IL}-1 \alpha$
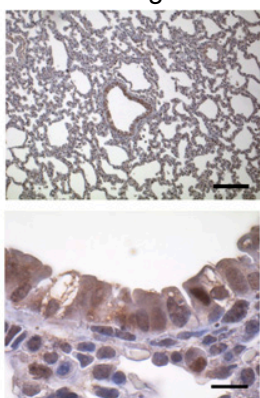

Figure 2. Localization of IL-1 $\alpha$ in lungs from neonatal wild-type (WT) and Scnn1b-Tg mice. (A) In situ hybridization of lung sections from neonatal ( $5 \mathrm{~d}$ old) WT and Scnn1b-Tg mice with locked nucleic acidmodified sense and antisense IL- $1 \alpha$ mRNA detection probes. Representative localization of IL-1 $\alpha$ transcripts (dark blue) in airway and lung parenchymal cells in WT and Scnn1b-Tg lungs hybridized with antisense probe. Staining was not detected in lung sections hybridized with sense probes. Scale bars $=100 \mu \mathrm{m}$ (top) and $10 \mu \mathrm{m}$ (bottom). (B) Immunohistochemical detection of IL-1 $\alpha$ in lung sections from neonatal WT and Scnn1b-Tg mice. Representative localization of IL-1 $\alpha$ (brown) in cytoplasm and nuclei of airway epithelial cells and some parenchymal cells in WT and Scnn1b-Tg lungs stained with primary anti-IL-1 $\alpha$ antibody $(I L-1 \alpha)$. Staining was not detected in lung sections incubated with secondary antibody only (control). All sections were counterstained with hematoxylin. Scale bars = $100 \mu \mathrm{m}$ (top) and $10 \mu \mathrm{m}$ (bottom). Representative of $\mathrm{n}=3-4$ mice per group from six litters.

necrotic cell death, modulated early mucus obstruction in neonatal Scnn $1 b$-Tg mice (Figure 5C). Collectively, these results demonstrate that IL-1R signaling is implicated in airway mucus obstruction and associated mortality in Scnn $1 b$-Tg mice, and suggest IL-1Ra as a potential modifier of mucus plugging.

\section{Lack of IL-1R Reduces Emphysema in Scnn1b-Tg Mice}

Chronic neutrophilic inflammation is associated with emphysema in Scnn $1 b$-Tg mice $(20,30)$. To determine if IL-1R signaling is implicated in the in vivo pathogenesis of emphysema, we compared mean linear intercepts as a measure of distal airspace enlargement and destructive index as a measure of alveolar wall destruction in 6-week-old Scnn1b-Tg/ Ill $r 1^{-/-}$mice with Scnn1b-Tg, Illr1 $1^{-/-}$ and WT littermates. As expected from previous studies, mean linear intercepts and destructive index were significantly increased in Scnn1b-Tg mice compared with WT (Figure 6). In Scnn1b-Tg/Il1r1 mice, emphysema parameters were significantly reduced compared with Scnn1b-Tg mice (Figure 6B). These results suggest an important role of IL-1R signaling in structural lung damage characteristic of chronic lung disease in Scnn $1 b$-Tg mice.

\section{Pharmacologic Inhibition of IL-1R Signaling Reduces Neutrophilic Airway Inflammation and Emphysema in Adult Scnn1b-Tg Mice}

To determine effects of pharmacologic inhibition of IL-1R signaling, we treated adult (4-6 wk old) Scnn1b-Tg mice and WT littermates by subcutaneous injections of the IL-1Ra anakinra for a period of 7 days. Similar to genetic deletion of IL-1R, treatment with anakinra had no effects on epithelial necrosis, but abrogated airway neutrophilia almost completely and reduced $\mathrm{KC}$ levels in BAL significantly in Scnn1b-Tg mice (Figures 7A-7C).
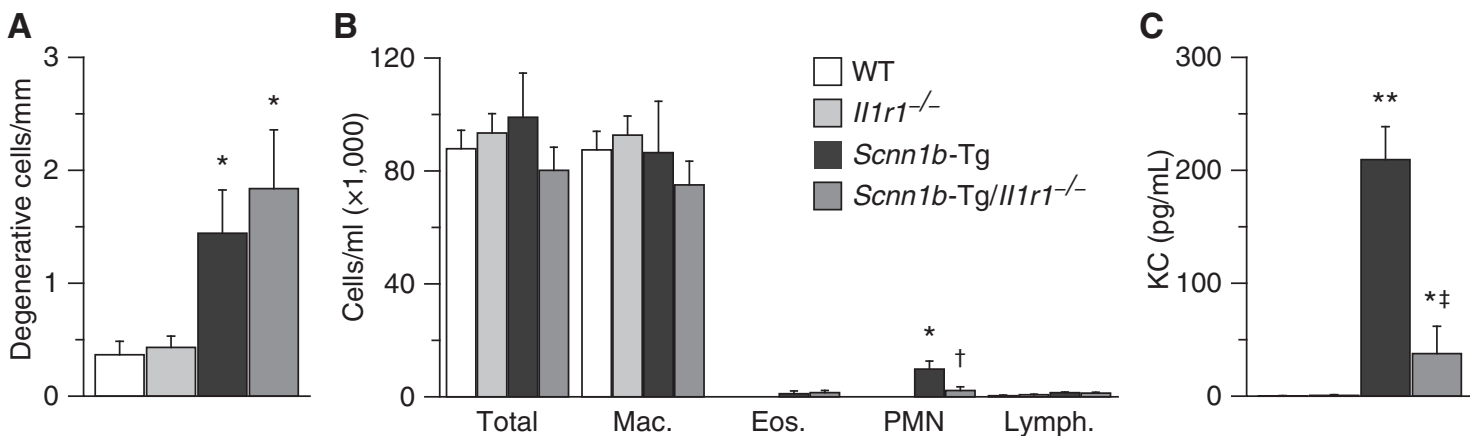

Figure 3. Lack of IL-1R reduces neutrophilic airway inflammation in adult Scnn1b-Tg mice. Comparison of airway epithelial necrosis $(A)$, bronchoalveolar lavage $(\mathrm{BAL})$ inflammatory cell counts $(B)$, and keratinocyte chemoattractant $(\mathrm{KC})$ levels $(C)$ in 6-week-old wild-type $(\mathrm{WT})$, $/ 11 \mathrm{r} 1^{-1-}$, Scnn $1 \mathrm{~b}$-Tg, and Scnn $1 \mathrm{~b}-\mathrm{Tg} / / 11 \mathrm{r} 1^{-1-}$ mice. (A) Summary of degenerative epithelial cells per millimeter of basement membrane. $\mathrm{n}=5-6 \mathrm{mice}$ per group from 14 litters. ${ }^{*} P<0.05$ compared with WT mice. ( $B$ and $C$ ) Inflammatory cell counts $(B)$ and concentration of $K C(C)$ in BAL. $n=4-13$ mice per group from 11-15 litters. ${ }^{\star} P<0.01$ and ${ }^{\star \star} P \leqslant 0.001$ compared with WT mice. ${ }^{\dagger} P<0.01$ and ${ }^{\ddagger} P \leqslant 0.001$ compared with Scnn $1 b$-Tg mice. Eos. = eosinophils; Lymph. $=$ lymphocytes; Mac. = macrophages; $\mathrm{PMN}=$ neutrophils. 

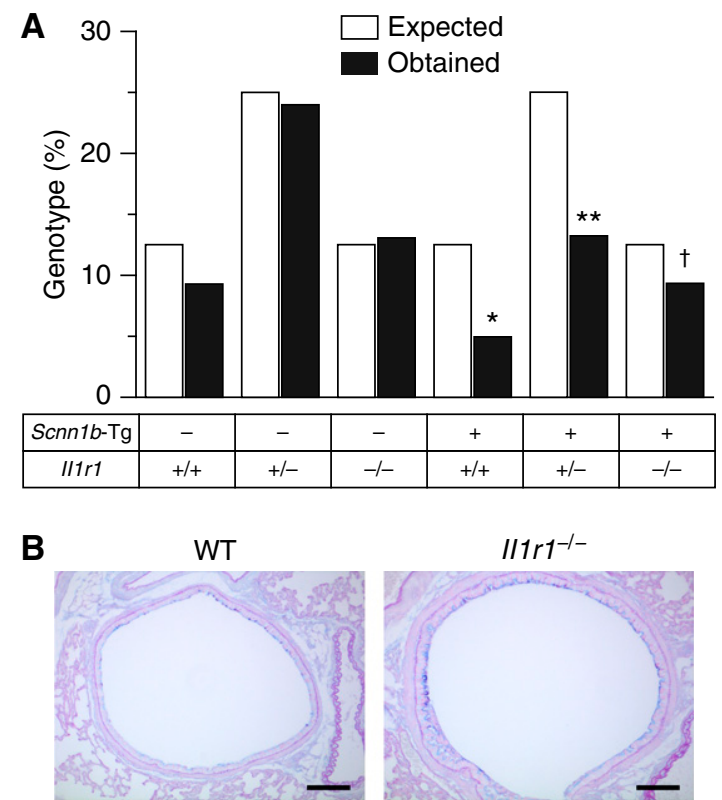

Scnn $1 b-\mathrm{Tg}$
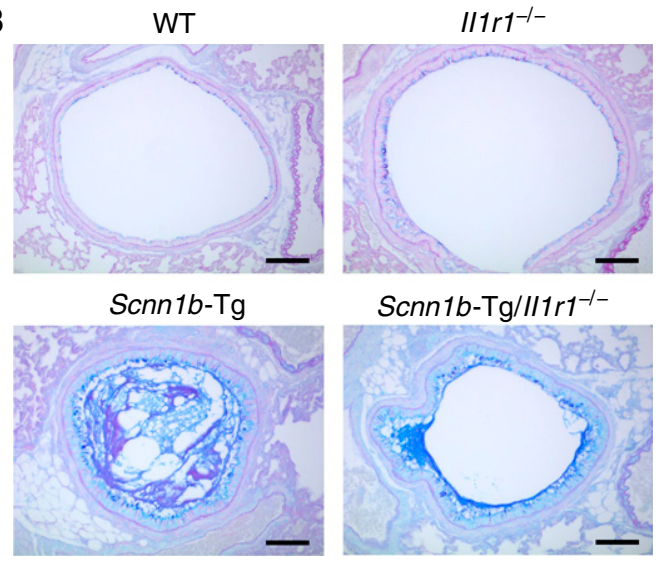

Scnn $1 b-\mathrm{Tg} / / 1111^{-1-}$
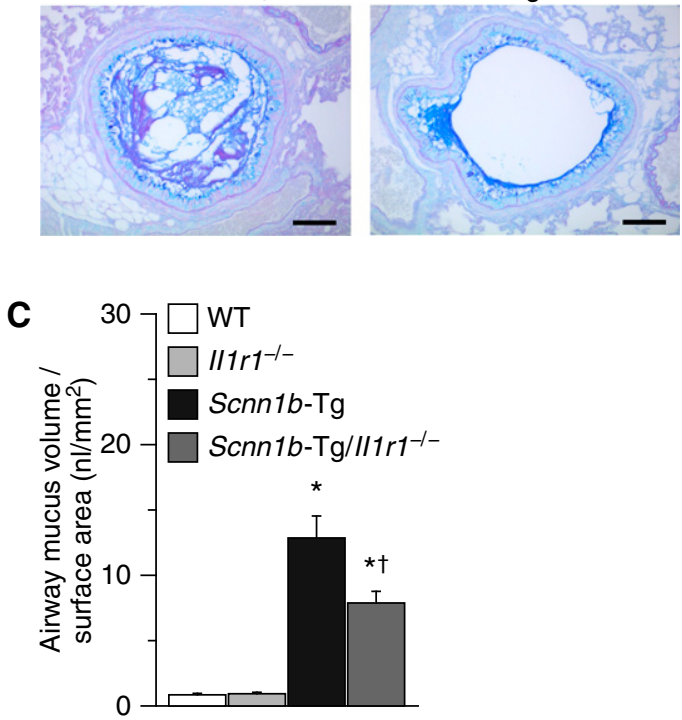

Figure 4. Lack of IL-1R reduces mortality and airway mucus obstruction in Scnn1b-Tg mice. Comparison of survival $(A)$ and airway mucus obstruction $(B$ and $C$ ) in 6-week-old wild-type (WT), $\| 11 r 1^{-1-}$, Scnn $1 b-\mathrm{Tg}$, and Scnn $1 \mathrm{~b}-\mathrm{Tg} / \| 1 \mathrm{r} \mathrm{r}^{-1-}$ mice. (A) Frequencies of expected versus obtained genotypes of mice derived from the intercross of Scnn $1 \mathrm{~b}$-Tg with $/ 11 \mathrm{r}^{-1-}$ mice that survived until the age of 6 weeks. $n=32-154$ mice per group from 66 litters. ${ }^{*} P<0.05$ for expected versus obtained genotype frequencies in Scnn1b-Tg compared with WT mice. ${ }^{\star \star} P \leqslant 0.001$ for expected versus obtained genotype frequencies in Scnn $1 b-\mathrm{Tg} / / 11 \mathrm{r} 1^{-/-}$compared with $/ 11 r 1^{+/-}$mice. ${ }^{\dagger} P<0.05$ for expected versus obtained genotype frequencies in Scnn $1 b-\mathrm{Tg} / / 11 \mathrm{r} 1^{-/-}$compared with Scnn $1 \mathrm{~b}-\mathrm{Tg}$ mice. $(B)$ Representative sections of proximal main axial airways stained with alcian blue periodic acid-Schiff. Scale bar $=100 \mu \mathrm{m}$. (C) Airway mucus content determined from the presence of alcian blue periodic acid-Schiff-positive material in airways from 6-week-old mice. $n=20-28$ mice per group from 23 litters. ${ }^{*} P \leqslant 0.001$ compared with WT mice. ${ }^{\dagger} P<0.01$ compared with $S c n n 1 b-\mathrm{Tg}$ mice.

Furthermore, anakinra treatment reduced emphysema, as determined from measurements of mean linear intercepts and the destructive index (Figures 7D and
7E; see Figure E2). In addition, we observed a trend toward reduction of airway mucus obstruction $(\sim 40 \% ; P=0.058)$ in anakinratreated versus vehicle-treated Scnn $1 b$-Tg mice (Figure 7F). These results demonstrate that pharmacologic inhibition of IL-1R signaling reduces neutrophilic inflammation and associated structural lung damage in adult Scnn1b-Tg mice with chronic lung disease.

\section{Epithelial Degeneration Is Present in Mucus-obstructed Airways of Patients with CF and COPD}

Our studies in mice with CF-like lung disease indicate that epithelial necrosis in mucus-obstructed airways may play an important role in the in vivo pathogenesis of neutrophilic inflammation. However, the occurrence of necrotic epithelial cells in airways from patients with $\mathrm{CF}$ and other chronic obstructive lung diseases remains unknown. We therefore evaluated sections of small airways ( $<2 \mathrm{~mm}$ diameter) from patients with CF or COPD and nonsmoking control subjects for the presence of degenerative epithelial cells (Figures 8A and 8C) and airway mucus (Figures 8A and 8B). Hydropic, highly vacuolated degenerative epithelial cells similar to the cells observed in Scnn1b-Tg mice (Figure 1A) were rarely observed in control subjects, but were significantly increased in mucus-obstructed airways from patients with CF (Figures $8 \mathrm{~A}$ and $8 \mathrm{C}$ ). Similarly, numeric densities of degenerative cells were increased in COPD compared with control subjects (Figures $8 \mathrm{~A}$ and $8 \mathrm{C}$ ). Of note, the number of necrotic airway epithelial cells was significantly correlated $(r=0.53 ; P<0.01)$ with the severity of airway mucus obstruction (Figure 8D). These results provide initial evidence that epithelial necrosis may be implicated in the pathogenesis of CF and potentially other chronic obstructive lung diseases in humans.

\section{Discussion}

Neutrophilic airway inflammation plays a key role in the onset and progression of lung disease in CF and other chronic obstructive lung diseases, even in the apparent absence of bacterial infection $(1,4,5,9,31-33)$. However, stimuli other than infection that can trigger airway neutrophilia remain poorly understood and therapeutic benefits of current antiinflammatory and antiinfective therapies remain limited. The importance of neutrophilic inflammation in early CF 
A

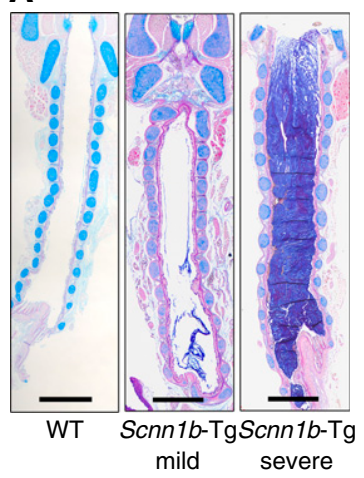

B

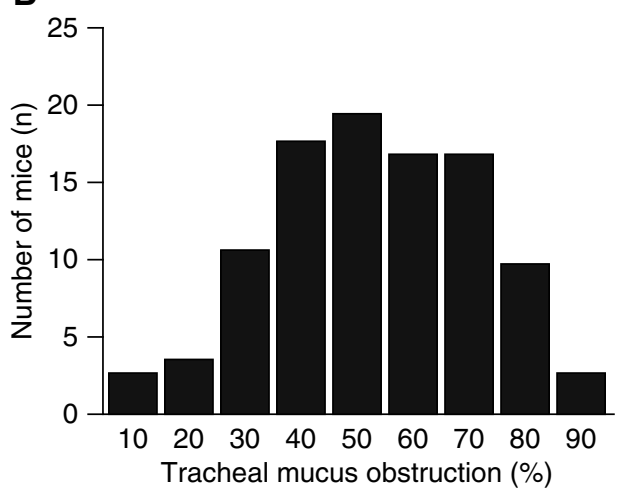

C

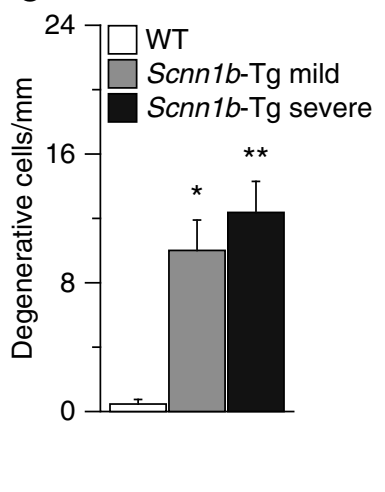

D

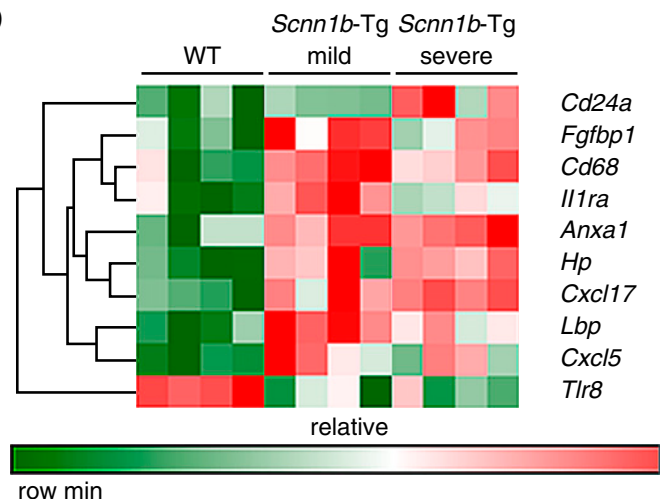

E

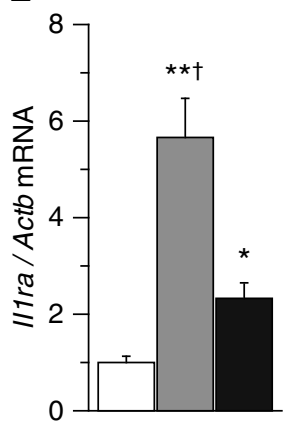

Figure 5. Severity of lung disease is associated with IL-1Ra transcript levels in neonatal Scnn1b-Tg mice. (A-C) Quantification of tracheal mucus obstruction and microarray gene expression analysis of 3-day-old Scnn1b-Tg and wild-type (WT) mice maintained on a mixed genetic background (C57BL/6N $\times \mathrm{C} 3 \mathrm{H} / \mathrm{HeN}$ ). (A) Representative sections of trachea from a 3-day-old WT mouse, a Scnn1b-Tg mouse with mild mucus obstruction, and a Scnn1b-Tg mouse with severe mucus obstruction stained with alcian blue periodic acid-Schiff. Scale bar $=500 \mu \mathrm{m}$. (B) Distribution of severity of tracheal mucus obstruction as determined from the percentage of the cross-sectional area of the tracheal lumen obstructed with alcian blue periodic acid-Schiff-positive material in 3-day-old Scnn1b-Tg mice. $n=102$ mice from 31 litters. (C) Summary of degenerative epithelial cells per millimeter of the basement membrane in airways from WT mice and Scnn $1 \mathrm{~b}$-Tg mice with mild ( $\leqslant 20 \%$ of luminal area) or severe ( $\geqslant 80 \%$ of luminal area) mucus obstruction. $\mathrm{n}=6-15$ mice per group from 15 litters. ${ }^{*} P<0.01$ and ${ }^{* *} P \leqslant 0.001$ compared with WT mice. (D) Heat map of genes related to inflammation that showed significant changes in expression in WT mice compared with Scnn $1 \mathrm{~b}$-Tg mice with mild ( $\leqslant 20 \%$ of luminal area) or severe $(\geqslant 80 \%$ of luminal area) mucus obstruction. $\mathrm{n}=4$ mice per group. (E) Quantitative real-time reverse transcriptase polymerase chain reaction for $\| 1$ ra expression in lungs from WT mice, Scnn1b-Tg mice with mild mucus obstruction, and Scnn1b-Tg mice with severe mucus obstruction. $n=6-7$ mice per group from 10 litters. ${ }^{\star} P<0.01$ and ${ }^{\star \star} P \leqslant 0.001$ compared with WT mice. ${ }^{\dagger} P<0.01$ compared with severely obstructed Scnn $1 b-\operatorname{Tg}$ mice.

pathogenesis was buttressed by recent studies demonstrating that airway neutrophilia and mucus obstruction are already present in infants with $\mathrm{CF}$, mostly in the absence of detectable bacterial infection $(1,2)$. However, these observational studies in infants with CF did not permit determination of whether inflammation occurred before or after the onset of mucus obstruction, and what stimuli trigger early inflammation in the absence of infection.

In previous longitudinal studies in Scnn1b-Tg mice with CF-like lung disease, we demonstrated that airway mucus plugging caused by increased absorption of $\mathrm{Na}^{+}$and liquid from airway surfaces was associated with cellular hypoxia and necrosis of a subset of airway Clara cells, as evidenced by in vivo labeling with the hypoxia probe pimonidazole hydrochloride and transmission electron microscopy studies showing intracellular glycogen depletion, endoplasmic recticulum (ER) vacuolarization, and pyknotic nuclei as sign of necrosis (20). Necrotic cells were observed from the first days of life before the onset of neutrophilic inflammation, whereas chronic airway neutrophilia with elevated levels of the neutrophil chemoattractant $\mathrm{KC}$, the functional ortholog of IL-8 in humans, was detected from postnatal day 5 onward $(20,21)$. Interestingly, neutrophilic inflammation was also observed in Scnn $1 b$-Tg mice raised in germ-free conditions demonstrating that inflammation occurred even with greatly reduced levels of pathogen-associated molecular patterns that activate MyD88 signaling via TLR (34).

Based on these results, and reports that activation of MyD88 signaling via IL-1R on nonhematopoietic cells by IL- $1 \alpha$ secreted in response to necrotic cell death constitutes a potent stimulus of sterile neutrophilic inflammation in other organs $(12,13)$ we investigated the role of this pathway in the development of airway neutrophilia in Scnn $1 b$-Tg mice. We found that genetic deletion of IL-1R had no effect on elevated necrotic airway epithelial cells and IL- $1 \alpha$, but abrogated airway neutrophilia and reduced $\mathrm{KC}$ in 5-day-old neonatal Scnn1bTg mice (Figure 1). Besides IL- $1 \alpha$ that is 
A

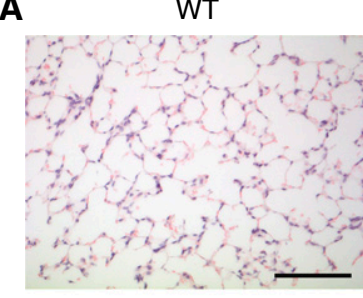
Scnn $1 b-\mathrm{Tg}$
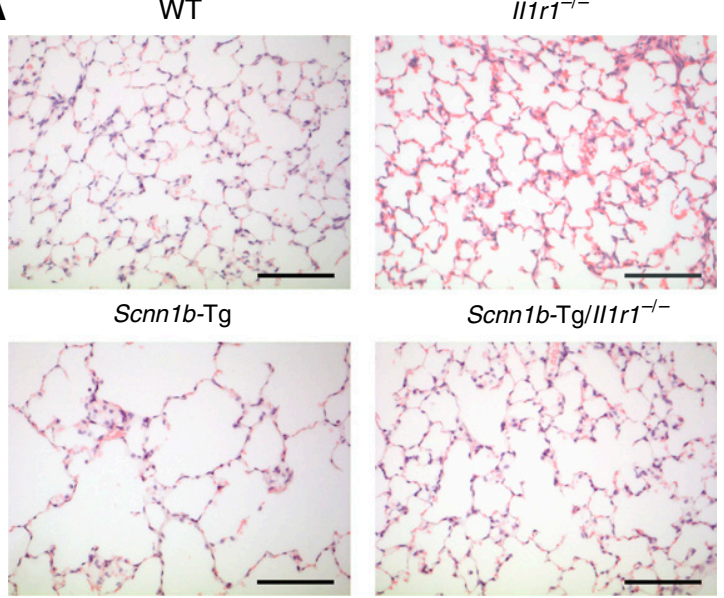

Scnn $1 b-\mathrm{Tg} / 1 / 1 r 1^{-1-}$

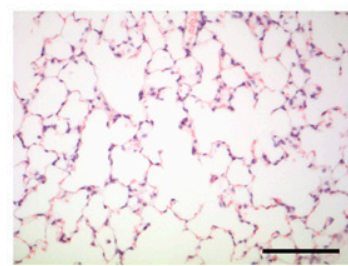

B
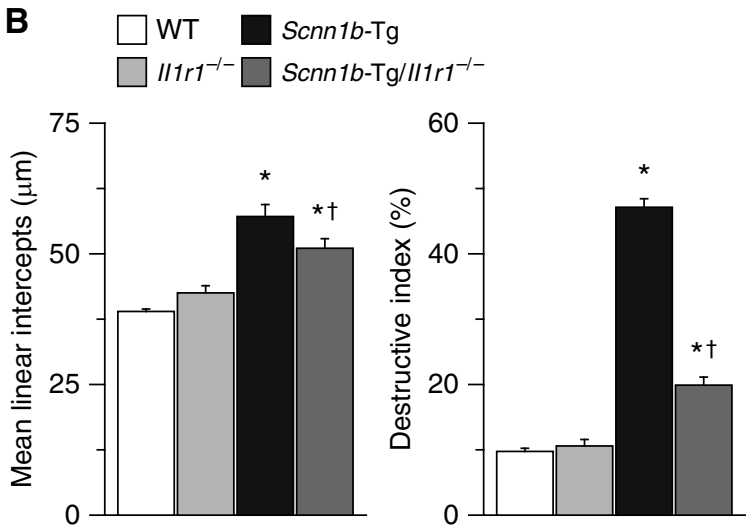

Figure 6. Lack of IL-1R reduces emphysema in Scnn1b-Tg mice. Comparison of distal airspace morphology $(A)$ and mean linear intercepts and destructive index $(B)$ in 6-week-old wild-type (WT), $\| 1 r 1^{-1-}$, Scnn $1 b-\mathrm{Tg}$, and Scnn $1 b-\mathrm{Tg} / \| 1 r 1^{-/-}$mice. (A) Representative lung sections stained with hematoxylin and eosin. Scale bars $=100 \mu \mathrm{m}$. (B, left) Summary of mean linear intercepts. $n=17-19$ mice per group from 27 litters. ${ }^{*} P \leqslant 0.001$ compared with WT mice. ${ }^{\dagger} P<0.05$ compared with Scnn1b-Tg mice. ( $B$, right) Destructive index. $\mathrm{n}=6$ mice per group from 13 litters. ${ }^{\star} P \leqslant 0.001$ compared with WT mice. ${ }^{\dagger} P \leqslant 0.001$ compared with Scnn $1 b$-Tg mice.

released in a fully functional form, IL-1R is activated by IL- $1 \beta$ that requires caspase1 -dependent activation of its inactive precursor in the inflammasome (15). In addition to neutrophils and KC, IL-1 $\beta$ was elevated in 5-day-old Scnn1b-Tg mice, but reduced to near normal levels in Scnn1b$\mathrm{Tg} / \operatorname{Il} 1 \mathrm{rl}^{-/-}$mice (Figure 1), indicating that secretion of IL-1 $\beta$ occurred secondary to IL- $1 \alpha$-mediated activation of IL1R-MyD88 signaling and inflammation. Tissue localization by in situ hybridization and immunohistochemistry showed distinct signals for IL- $1 \alpha$ transcripts and protein in the airway epithelium and to a lesser extent in lung parenchyma, but not in macrophages contained in lung sections from WT and Scnn1b-Tg mice (Figure 2; see Figure E1). As observed in neonatal mice, necrotic airway epithelial cells remained elevated, but airway neutrophilia and $\mathrm{KC}$ were significantly reduced in adult Scnn1b-Tg/Il1r1 ${ }^{-/-}$compared with Scnn1b-Tg mice (Figure 3). Collectively, these results identify activation of IL-1RMyD88 signaling by IL- $1 \alpha$ that is released from airway epithelial cells undergoing necrosis, rather than infiltrating macrophages responding to epithelial injury, as an important pathway in the onset and perpetuation of neutrophilic inflammation in mice with CF-like lung disease.

Our studies also demonstrate that IL1R-MyD88 pathway activation by epithelial necrosis contributes to CF-like airway mucus obstruction in Scnn $1 b$-Tg mice. Previous longitudinal studies demonstrated that a subgroup of Scnn1b-Tg mice die in the neonatal period because of severe mucus plugging of the trachea and that adult survivors exhibit chronic mucus obstruction of intrapulmonary airways. Consistent with a role of IL-1R-MyD88 signaling in airway mucus obstruction in the present study, survival was significantly increased and airway mucus content was reduced in adult Scnn $1 b-\mathrm{Tg} / \mathrm{Il} 1 \mathrm{r} 1^{-/-}$ compared with Scnn1b-Tg mice (Figure 4). The importance of IL-1R-MyD88 signaling in early mucus obstruction was supported by independent studies using wholegenome expression profiling of lungs from neonatal Scnn1b-Tg mice on a mixed genetic background with variable mucus obstruction (20) that identified an inverse relationship between the severity of tracheal mucus plugging and expression of Ill ra (Figure 5). Of note, previous studies from various laboratories showed that activation of MAPK and nuclear factor- $\kappa \mathrm{B}$ via TLRMyD88 signaling induces mucin expression in mice and human airway cultures (35-37) establishing a role of this pathway in mucus hypersecretion associated with airway infection and inflammation. Furthermore, it was shown that genetic deletion of the adaptor molecule MyD88 reduced goblet cell metaplasia and airway mucus plugging in neonatal Scnn1b-Tg mice in the context of bacterial airway infection. However, whether MyD88 was activated via TLRs or IL-1R remained unknown (34).

We recently demonstrated that lack of NE, another potent stimulus of mucus hypersecretion (10), reduced goblet cell metaplasia and reduced elevated mucin expression. However, in contrast to genetic deletion of Il1r, lack of NE was not sufficient to prevent airway mucus obstruction and associated mortality in Scnn1b-Tg mice (9). Based on these results, we speculate that activation of the IL$1 \mathrm{R}-\mathrm{MyD} 88$ pathway by IL- $1 \alpha$ released from necrotic epithelial cells provides an alternative stimulus of mucin hypersecretion that may aggravate mucus hyperconcentration and plugging independent of pathogens and NE. In addition, these results suggest that the endogenous inhibitor of this pathway, IL$1 \mathrm{Ra}$, may act not only as a modulator of inflammation, but also as a potential modifier of mucus hypersecretion and obstruction. However, in contrast to previous studies where preventive treatment with the epithelial sodium channel blocker amiloride abrogated airway mucus obstruction almost completely and reduced necrotic cells substantially 
A

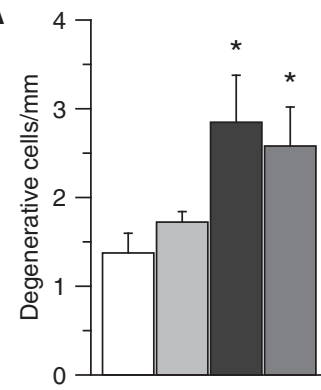

B

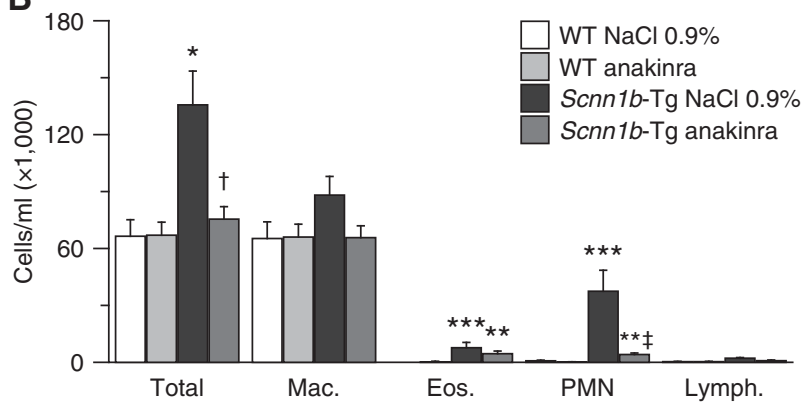

C

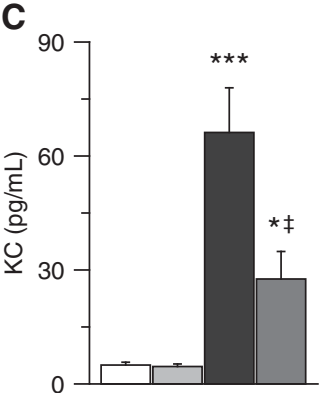

D

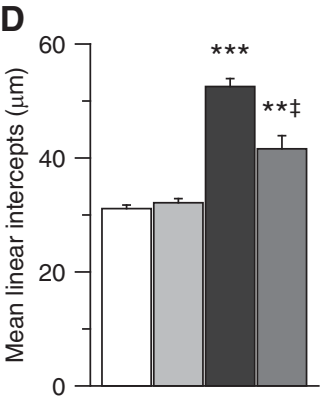

E

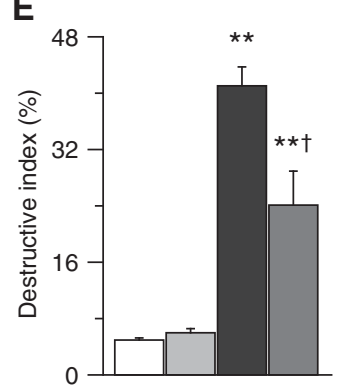

$\mathbf{F}$

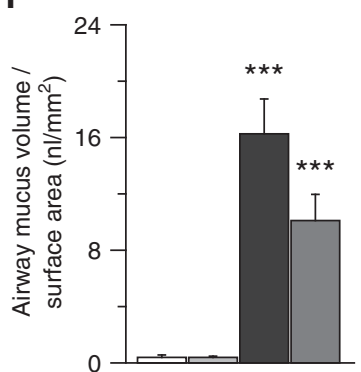

Figure 7. Anakinra treatment reduces neutrophilic airway inflammation and emphysema in Scnn1b-Tg mice. Adult (4-6 wk old) wild-type (WT) and Scnn $1 \mathrm{~b}$-Tg mice were treated with subcutaneous injection of anakinra (200 mg/kg body weight) or vehicle ( $\mathrm{NaCl} 0.9 \%)$ alone twice per day for 7 days. Comparison of airway epithelial necrosis $(A)$, bronchoalveolar lavage $(\mathrm{BAL})$ inflammatory cell counts $(B)$, and keratinocyte chemoattractant $(\mathrm{KC})$ levels (C). (A) Summary of degenerative epithelial cells per millimeter of basement membrane. $n=6-9$ mice per group. (B) Inflammatory cell counts and (C) concentrations of KC in BAL. $n=11-16$ mice per group. ( $D$ and $E$ ) Comparison of mean linear intercepts $(D)$ and destructive index $(E)$. $n=6-9$ mice per group. ( $F$ ) Airway mucus content determined from the presence of alcian blue periodic acid-Schiff-positive material in airways. $n=14-16$ mice per group. ${ }^{\star} P<0.05,{ }^{\star \star} P<0.01$, and ${ }^{* \star} P \leqslant 0.001$ compared with vehicle-treated WT mice. ${ }^{\dagger} P<0.05$ and ${ }^{\ddagger} P<0.01$ compared with vehicle-treated Scnn $1 b$-Tg mice. Data were derived from four independent studies with mice from 14 litters. Eos. = eosinophils; Lymph. = lymphocytes; Mac. = macrophages; PMN = neutrophils.

$(\sim 50 \%)$ (22), neither genetic deletion nor pharmacologic inhibition of IL-1R signaling had effects on epithelial necrosis in Scnn $1 b$ Tg mice (Figures 1, 3, 7). We therefore speculate that the moderate $(\sim 40 \%)$ reduction in airway mucus content obtained by inhibition of IL-1R signaling was not sufficient to improve luminal $\mathrm{O}_{2}$ delivery to levels that result in a measurable reduction of epithelial necrosis in the presence of CF-like $\mathrm{Na}^{+}$hyperabsorption that has been reported to increase epithelial $\mathrm{O}_{2}$ consumption $(16,20)$.

Neutrophilic airway inflammation constitutes a major risk factor for early onset and progression of bronchiectasis in patients with $\mathrm{CF}$ and is associated with early structural damage of distal airspaces in Scnn $1 b$-Tg mice $(1,4,20,30)$. The present study demonstrates that genetic deletion of the IL-1R-MyD88 pathway resulted in a significant reduction of structural lung damage in this model of CF lung disease (Figure 6). In recent studies, we demonstrated that proteolytic activities of NE and matrix metalloproteinase 12 released from activated neutrophils and macrophages in chronic airway inflammation play key roles in emphysema formation in Scnn1b-Tg mice $(9,27)$. We therefore speculate that the abrogation of airway neutrophilia reduced elastolytic activity, thus ameliorating structural lung damage in Scnn1b-Tg/Il1r1 $1^{-/-}$mice (Figure 6). However, infiltration of airspaces by enlarged, morphologically activated macrophages persisted (Figure 1C) suggesting that secretion of matrix metalloproteinase 12 may contribute to residual lung damage observed in Scnn1b-Tg/Il1r1 $1^{-/-}$mice (Figure 6).

Hypoxia is a common feature in $\mathrm{CF}$ lungs that is caused by airway mucus plugging and increased epithelial oxygen consumption due to increased epithelial sodium channel-mediated absorption of $\mathrm{Na}^{+}$and fluid from airway surfaces (16, 17). The role of hypoxia in CF has been studied in the context of chronic infection with Pseudomonas aeruginosa and anaerobic bacteria $(17,38)$. However, although it is well established that hypoxic necrosis is a potent stimulus of neutrophilic inflammation in other common diseases, such as stroke and myocardial infarction (12), the prevalence of necrotic cells in CF airways remains unknown. To determine the relevance of our findings in Scnn $1 b$-Tg mice for CF lung disease in humans, we evaluated sections of small airways from patients with CF for the presence of necrotic cells. We show for the first time that hydropic degeneration (i.e., the morphologic correlate of necrotic cell death [39]), was detectable in the airway epithelium and significantly increased ( $\sim 2.3$-fold) in patients with CF compared with control subjects (Figure 8).

Based on recent evidence suggesting that patients with COPD exhibit acquired CF transmembrane conductance regulator (CFTR) dysfunction leading to CF-like dehydration of airway surfaces (40-43) and that small airways mucus plugging also plays an important role in COPD (44), we extended our studies to patients with this common chronic obstructive lung disease. Similar to CF, small airways from patients with COPD exhibited intraluminal mucus obstruction and increased numbers of highly vacuolated degenerative epithelial cells. 
A
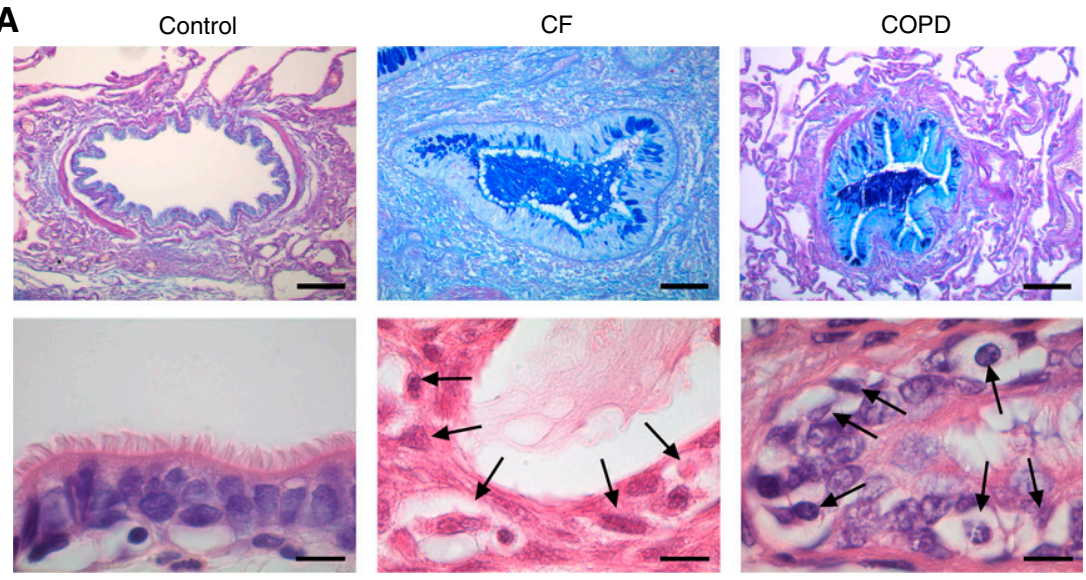

B

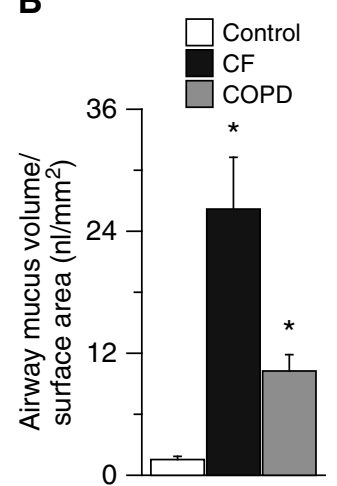

C

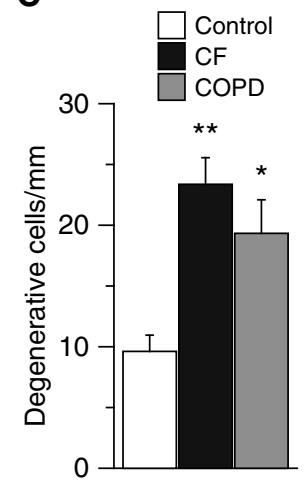

D

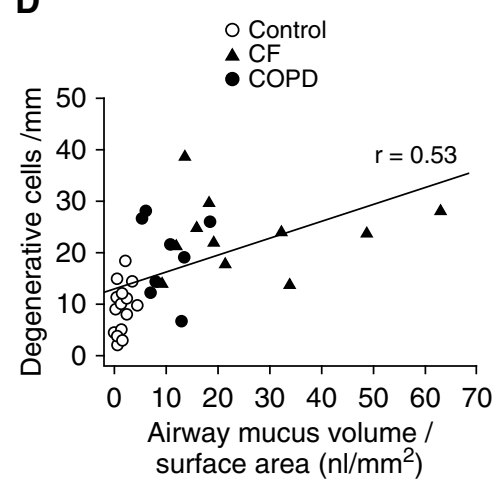

Figure 8. Mucus obstruction is associated with epithelial necrosis in small airways of patients with cystic fibrosis (CF) and chronic obstructive pulmonary disease (COPD). (A) Representative airway sections from a control subject and patients with CF and COPD stained with alcian blue periodic acid-Schiff to determine the presence of mucus (top, scale bars $=100 \mu \mathrm{m}$ ) and hematoxylin and eosin to identify degenerative airway epithelial cells (arrows) (bottom, scale bars $=10 \mu \mathrm{m})$. (B and $C)$ Comparison of airway mucus content determined from the presence of alcian blue periodic acid-Schiff-positive material $(B)$ and numeric densities of degenerative airway epithelial cells in control, CF, and COPD groups $(C) . n=8-19$ individuals per group. ${ }^{*} P<0.01$ and ${ }^{* *} P<0.001$ compared with control subjects. $(D)$ Correlation between numeric densities of degenerative cells and airway mucus content in control subjects and patients with CF and COPD. $n=8-15$ individuals per group. Pearson product-moment correlation coefficient $r=0.53, P<0.01$.

Furthermore, we found that necrotic cell densities correlated with airway mucus content in CF and COPD (Figure 8). Taken together, these results show that small airways of patients with CF and COPD exhibit increased frequencies of necrotic epithelial cells in situ suggesting that release of IL- $1 \alpha$ from necrotic cells may activate IL-1R-MyD88 signaling and contribute to neutrophilic airway inflammation characteristic of these chronic obstructive lung diseases.

Our preclinical studies with the IL-1Ra anakinra show that pharmacologic inhibition of IL-1R signaling had significant protective effects in adult Scnn $1 b$-Tg mice with chronic lung disease (Figure 7; see
Figure E2). Similar to genetic deletion of IL$1 \mathrm{R}$, anakinra treatment abrogated airway neutrophilia almost completely and reduced KC levels significantly in Scnn 1 b-Tg mice demonstrating potent antiinflammatory effects. Of note, inhibition of airway neutrophilia by anakinra treatment was associated with a significant reduction of distal airspace enlargement and structural damage characteristic of Scnn $1 b-\mathrm{Tg}$ mice (20). These results are consistent with an important role of NE in emphysema formation in Scnn1b-Tg mice (9), and work from other laboratories demonstrating substantial neoalveolarization during compensatory lung growth after pneumonectomy or elastase-induced emphysema in mice $(45,46)$.

Taken together, these results support inhibition of IL-1R signaling as a promising antiinflammatory strategy. However, therapeutic strategies that inhibit neutrophilic inflammation to prevent lung damage also have the potential risk to further compromise antibacterial host defense and aggravate infection-related exacerbations in CF (47). Because TLRMyD88 signaling remains intact even when the IL-1R-MyD88 pathway is blocked, therapeutic targeting of IL-1R may provide an attractive strategy to reduce CF airways inflammation without disabling host defense against pathogens. Consistent with this concept, previous studies showed that recruitment of neutrophils by microbial stimuli was not impaired in $I l 1 \mathrm{rl}^{-/-}$mice and patients treated with IL-1 blocking agents for other inflammatory disorders have not shown an increased risk of opportunistic infections $(12,15)$. Although genetic deletion and pharmacologic inhibition of IL-1R reduced influx of neutrophils, recruitment of and morphologic activation of macrophages remained intact in Scnn1b-Tg mice (Figure 1C) and in mice with necrosisinduced inflammation in other organs (12).

We therefore speculate that therapeutic inhibition of IL-1R signaling may have benefits by limiting the damaging effects of airway neutrophils (Figure 7) $(4,8,9,31)$ without compromising antibacterial host defense or macrophage-mediated tissue repair functions in CF airways. In addition to anakinra, several other agents that block IL-1 signaling including soluble decoy receptor and IL-1 $\beta$ neutralizing antibodies have been approved for the treatment of other chronic inflammatory diseases (15), and these existing drugs may facilitate clinical testing of benefits of IL-1R pathway blockade in CF and potentially other chronic neutrophilic airway diseases.

In addition to the Scnn $1 b$-Tg mouse, CFTR-deficient pigs and ferrets have recently become available for in vivo studies of the relationship between mucus, inflammation, and infection in early $\mathrm{CF}$ lung disease $(48,49)$. In newborn CF pigs, lack of CFTR was shown to cause impaired mucociliary clearance and a $\mathrm{pH}$-dependent defect in bacterial killing in the absence of detectable airway surface liquid depletion and inflammation $(48,50,51)$. In newborn CF ferrets, CFTR malfunction was shown 
to produce airway surface liquid depletion and elevated IL-8 levels in BAL even in the absence of detectable bacteria. CF ferrets also showed a defect in bacterial killing following challenge with $P$. aeruginosa. However, this defect was not $\mathrm{pH}$-dependent (52). Despite these distinct differences in initial phenotypes, both models develop a spontaneous lung disease that shares key features of lung disease in infants and young children with $\mathrm{CF}(1,2,53)$. These include regions with highly tenacious mucus plugs that contain neutrophils and cause obstruction of airways and air trapping in otherwise unaffected lungs, and regions with a more purulent airway exudate and associated pneumonia $(48,49)$. We expect that these additional animal models will provide unique opportunities for further elucidation of the relative role of epithelial necrosis and IL-1R signaling in the complex in vivo pathogenesis of neutrophilic inflammation at different stages of CF lung disease.

In summary, this study demonstrates for the first time that necrosis of airway epithelial cells is a characteristic feature of CF lung disease in mice and patients that provides a potent stimulus of neutrophilic inflammation. Furthermore, we identified activation of IL-1R-MyD88 signaling by IL- $1 \alpha$ released from necrotic cells as the mechanism underlying necrosis-induced airway neutrophilia. Besides neutrophilic inflammation, we show that activation of the IL-1R-MyD88 pathway is implicated in mucus obstruction and structural lung damage in vivo. These results suggest that hypoxic epithelial necrosis may provide an important trigger for neutrophilic airway inflammation independent of bacterial infection, and support inhibition of IL-1R signaling as a novel antiinflammatory strategy in CF and potentially other mucoobstructive airway diseases.

Author disclosures are available with the text of this article at www.atsjournals.org.

Acknowledgment: The authors thank N. Gretz (Microarray Analysis Core Laboratory, Medical Research Center, Medical Faculty Mannheim, University of Heidelberg) for expert assistance with microarray analysis and S. Schmidt and E. Maier for expert technical assistance.

\section{References}

1. Sly PD, Brennan S, Gangell C, de Klerk N, Murray C, Mott L, Stick SM, Robinson PJ, Robertson CF, Ranganathan SC; Australian Respiratory Early Surveillance Team for Cystic Fibrosis (AREST-CF). Lung disease at diagnosis in infants with cystic fibrosis detected by newborn screening. Am J Respir Crit Care Med 2009;180:146-152.

2. Grasemann $\mathrm{H}$, Ratjen F. Early lung disease in cystic fibrosis. Lancet Respir Med 2013;1:148-157.

3. Voynow JA, Fischer BM, Zheng S. Proteases and cystic fibrosis. Int $J$ Biochem Cell Biol 2008;40:1238-1245.

4. Sly PD, Gangell CL, Chen L, Ware RS, Ranganathan S, Mott LS, Murray CP, Stick SM; AREST CF Investigators. Risk factors for bronchiectasis in children with cystic fibrosis. N Engl J Med 2013;368:1963-1970.

5. Sagel SD, Wagner BD, Anthony MM, Emmett P, Zemanick ET. Sputum biomarkers of inflammation and lung function decline in children with cystic fibrosis. Am J Respir Crit Care Med 2012;186:857-865.

6. Le Gars M, Descamps D, Roussel D, Saussereau E, Guillot L, Ruffin M, Tabary O, Hong SS, Boulanger P, Paulais M, et al. Neutrophil elastase degrades cystic fibrosis transmembrane conductance regulator via calpains and disables channel function in vitro and in vivo. $A m \mathrm{~J}$ Respir Crit Care Med 2013;187:170-179.

7. Caldwell RA, Boucher RC, Stutts MJ. Neutrophil elastase activates nearsilent epithelial $\mathrm{Na}+$ channels and increases airway epithelial $\mathrm{Na}+$ transport. Am J Physiol Lung Cell Mol Physiol 2005;288:L813-L819.

8. Taggart CC, Greene CM, Carroll TP, O'Neill SJ, McElvaney NG. Elastolytic proteases: inflammation resolution and dysregulation in chronic infective lung disease. Am J Respir Crit Care Med 2005;171: 1070-1076.

9. Gehrig S, Duerr J, Weitnauer M, Wagner CJ, Graeber SY, Schatterny J, Hirtz S, Belaaouaj A, Dalpke AH, Schultz C, et al. Lack of neutrophil elastase reduces inflammation, mucus hypersecretion, and emphysema, but not mucus obstruction, in mice with cystic fibrosislike lung disease. Am J Respir Crit Care Med 2014;189:1082-1092.

10. Voynow JA, Gendler SJ, Rose MC. Regulation of mucin genes in chronic inflammatory airway diseases. Am J Respir Cell Mol Biol 2006;34:661-665.

11. Moghaddam SJ, Clement CG, De la Garza MM, Zou X, Travis EL, Young HW, Evans CM, Tuvim MJ, Dickey BF. Haemophilus influenzae lysate induces aspects of the chronic obstructive pulmonary disease phenotype. Am J Respir Cell Mol Biol 2008;38: 629-638.

12. Chen CJ, Kono H, Golenbock D, Reed G, Akira S, Rock KL. Identification of a key pathway required for the sterile inflammatory response triggered by dying cells. Nat Med 2007;13:851-856.

13. Lukens JR, Gross JM, Kanneganti TD. IL-1 family cytokines trigger sterile inflammatory disease. Front Immunol 2012;3:315.
14. Watters TM, Kenny EF, O'Neill LA. Structure, function and regulation of the Toll/IL-1 receptor adaptor proteins. Immunol Cell Biol 2007;85:411-419.

15. Dinarello CA, Simon A, van der Meer JW. Treating inflammation by blocking interleukin-1 in a broad spectrum of diseases. Nat Rev Drug Discov 2012;11:633-652.

16. Stutts MJ, Knowles MR, Gatzy JT, Boucher RC. Oxygen consumption and ouabain binding sites in cystic fibrosis nasal epithelium. Pediatr Res 1986;20:1316-1320.

17. Worlitzsch D, Tarran R, Ulrich M, Schwab U, Cekici A, Meyer KC, Birrer $\mathrm{P}$, Bellon G, Berger J, Weiss T, et al. Effects of reduced mucus oxygen concentration in airway Pseudomonas infections of cystic fibrosis patients. J Clin Invest 2002;109:317-325.

18. Mall M, Grubb BR, Harkema JR, O'Neal WK, Boucher RC. Increased airway epithelial $\mathrm{Na}^{+}$absorption produces cystic fibrosis-like lung disease in mice. Nat Med 2004;10:487-493.

19. Zhou Z, Duerr J, Johannesson B, Schubert SC, Treis D, Harm M, Graeber SY, Dalpke A, Schultz C, Mall MA. The ENaCoverexpressing mouse as a model of cystic fibrosis lung disease. $J$ Cyst Fibros 2011;10:S172-S182.

20. Mall MA, Harkema JR, Trojanek JB, Treis D, Livraghi A, Schubert S, Zhou Z, Kreda SM, Tilley SL, Hudson EJ, et al. Development of chronic bronchitis and emphysema in $\beta$-epithelial $\mathrm{Na}^{+}$channeloverexpressing mice. Am J Respir Crit Care Med 2008;177:730-742.

21. Johannesson B, Hirtz S, Schatterny J, Schultz C, Mall MA. CFTR regulates early pathogenesis of chronic obstructive lung disease in

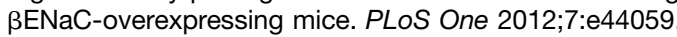

22. Zhou Z, Treis D, Schubert SC, Harm M, Schatterny J, Hirtz S, Duerr J, Boucher RC, Mall MA. Preventive but not late amiloride therapy reduces morbidity and mortality of lung disease in betaENaCoverexpressing mice. Am J Respir Crit Care Med 2008;178: 1245-1256.

23. Zhou-Suckow ZFB, Schatterny J, Hirtz S, Mall MA. Hypoxic epithelial necrosis, IL-1 receptor, siganling and neutrophilic airway inflammation in $\beta E N a C$-overexpressing mice [abstract]. Pediatr Pulmonol 2013;48:A181.

24. Vestbo J, Hurd SS, Agustí AG, Jones PW, Vogelmeier C, Anzueto A, Barnes PJ, Fabbri LM, Martinez FJ, Nishimura M, et al. Global strategy for the diagnosis, management, and prevention of chronic obstructive pulmonary disease: GOLD executive summary. Am J Respir Crit Care Med 2013;187:347-365.

25. Glaccum MB, Stocking KL, Charrier K, Smith JL, Willis CR, Maliszewski C, Livingston DJ, Peschon JJ, Morrissey PJ. Phenotypic and functional characterization of mice that lack the type I receptor for IL-1. J Immunol 1997;159:3364-3371.

26. Saetta M, Shiner RJ, Angus GE, Kim WD, Wang NS, King M, Ghezzo H, Cosio MG. Destructive index: a measurement of lung parenchymal destruction in smokers. Am Rev Respir Dis 1985;131:764-769. 
27. Trojanek JB, Cobos-Correa A, Diemer S, Kormann M, Schubert SC, Zhou-Suckow Z, Agrawal R, Duerr J, Wagner CJ, Schatterny J, et al. Airway mucus obstruction triggers macrophage activation and matrix metalloproteinase 12-dependent emphysema. Am J Respir Cell Mol Biol 2014;51:709-720.

28. Botelho FM, Bauer CM, Finch D, Nikota JK, Zavitz CC, Kelly A, Lambert KN, Piper S, Foster ML, Goldring JJ, et al. IL-1 $\alpha / \mathrm{IL}-1 \mathrm{R} 1$ expression in chronic obstructive pulmonary disease and mechanistic relevance to smoke-induced neutrophilia in mice. PLoS One 2011;6:e28457.

29. Kloosterman WP, Wienholds E, de Bruijn E, Kauppinen S, Plasterk RH. In situ detection of miRNAs in animal embryos using LNA-modified oligonucleotide probes. Nat Methods 2006;3:27-29.

30. Wielpütz MO, Eichinger M, Zhou Z, Leotta K, Hirtz S, Bartling SH, Semmler W, Kauczor HU, Puderbach M, Mall MA. In vivo monitoring of cystic fibrosis-like lung disease in mice by volumetric computed tomography. Eur Respir J 2011;38:1060-1070.

31. Hoenderdos K, Condliffe A. The neutrophil in chronic obstructive pulmonary disease. Am J Respir Cell Mol Biol 2013; 48:531-539.

32. Muhlebach MS, Noah TL. Endotoxin activity and inflammatory markers in the airways of young patients with cystic fibrosis. Am J Respir Crit Care Med 2002;165:911-915.

33. Berger M. Lung inflammation early in cystic fibrosis: bugs are indicted, but the defense is guilty. Am J Respir Crit Care Med 2002;165: 857-858.

34. Livraghi-Butrico A, Kelly EJ, Klem ER, Dang H, Wolfgang MC, Boucher RC, Randell SH, O'Neal WK. Mucus clearance, MyD88-dependent and MyD88-independent immunity modulate lung susceptibility to spontaneous bacterial infection and inflammation. Mucosal Immunol 2012;5:397-408.

35. Fujisawa T, Chang MM, Velichko S, Thai $P$, Hung LY, Huang F, Phuong N, Chen Y, Wu R. NF-кB mediates IL-1 $\beta$ - and IL-17A-induced MUC5B expression in airway epithelial cells. Am J Respir Cell Mol Biol 2011;45:246-252.

36. Chen Y, Garvin LM, Nickola TJ, Watson AM, Colberg-Poley AM, Rose MC. IL-1 $\beta$ induction of MUC5AC gene expression is mediated by CREB and NF- $\mathrm{BB}$ and repressed by dexamethasone. Am J Physiol Lung Cell Mol Physiol 2014;306:L797-L807.

37. Jono H, Xu H, Kai H, Lim DJ, Kim YS, Feng XH, Li JD. Transforming growth factor-beta-Smad signaling pathway negatively regulates nontypeable Haemophilus influenzae-induced MUC5AC mucin transcription via mitogen-activated protein kinase (MAPK) phosphatase-1-dependent inhibition of p38 MAPK. J Biol Chem 2003;278:27811-27819.

38. Tunney MM, Field TR, Moriarty TF, Patrick S, Doering G, Muhlebach MS, Wolfgang MC, Boucher R, Gilpin DF, McDowell A, et al. Detection of anaerobic bacteria in high numbers in sputum from patients with cystic fibrosis. Am J Respir Crit Care Med 2008;177: 995-1001.

39. Majno G, Joris I. Apoptosis, oncosis, and necrosis. An overview of cell death. Am J Pathol 1995;146:3-15.

40. Mall MA, Hartl D. CFTR: cystic fibrosis and beyond. Eur Respir J 2014; 44:1042-1054.
41. Cantin AM, Hanrahan JW, Bilodeau G, Ellis L, Dupuis A, Liao J, Zielenski J, Durie P. Cystic fibrosis transmembrane conductance regulator function is suppressed in cigarette smokers. Am J Respir Crit Care Med 2006;173:1139-1144.

42. Clunes LA, Davies CM, Coakley RD, Aleksandrov AA, Henderson AG Zeman KL, Worthington EN, Gentzsch M, Kreda SM, Cholon D, et al. Cigarette smoke exposure induces CFTR internalization and insolubility, leading to airway surface liquid dehydration. FASEB J 2012;26:533-545.

43. Dransfield MT, Wilhelm AM, Flanagan B, Courville C, Tidwell SL, Raju SV, Gaggar A, Steele C, Tang LP, Liu B, et al. Acquired cystic fibrosis transmembrane conductance regulator dysfunction in the lower airways in COPD. Chest 2013;144:498-506.

44. Hogg JC, Chu F, Utokaparch S, Woods R, Elliott WM, Buzatu L, Cherniack RM, Rogers RM, Sciurba FC, Coxson HO, et al. The nature of small-airway obstruction in chronic obstructive pulmonary disease. N Engl J Med 2004;350:2645-2653.

45. Fehrenbach H, Voswinckel R, Michl V, Mehling T, Fehrenbach A, Seeger W, Nyengaard JR. Neoalveolarisation contributes to compensatory lung growth following pneumonectomy in mice. Eur Respir J 2008;31:515-522.

46. Hoffman AM, Shifren A, Mazan MR, Gruntman AM, Lascola KM, NolenWalston RD, Kim CF, Tsai L, Pierce RA, Mecham RP, et al. Matrix modulation of compensatory lung regrowth and progenitor cell proliferation in mice. Am J Physiol Lung Cell Mol Physiol 2010;298: L158-L168.

47. Döring G, Bragonzi A, Paroni M, Aktürk FF, Cigana C, Schmidt A, Gilpin D, Heyder S, Born T, Smaczny C, et al. BIIL 284 reduces neutrophil numbers but increases $P$. aeruginosa bacteremia and inflammation in mouse lungs. J Cyst Fibros 2014;13:156-163.

48. Stoltz DA, Meyerholz DK, Pezzulo AA, Ramachandran S, Rogan MP, Davis GJ, Hanfland RA, Wohlford-Lenane C, Dohrn CL, Bartlett JA, et al. Cystic fibrosis pigs develop lung disease and exhibit defective bacterial eradication at birth. Sci Trans/ Med 2010;2:29ra31.

49. Sun X, Olivier AK, Liang B, Yi Y, Sui H, Evans TI, Zhang Y, Zhou W, Tyler SR, Fisher JT, et al. Lung phenotype of juvenile and adult cystic fibrosis transmembrane conductance regulator-knockout ferrets. $\mathrm{Am}$ $J$ Respir Cell Mol Biol 2014;50:502-512.

50. Hoegger MJ, Fischer AJ, McMenimen JD, Ostedgaard LS, Tucker AJ, Awadalla MA, Moninger TO, Michalski AS, Hoffman EA, Zabner J, et al. Cystic fibrosis. Impaired mucus detachment disrupts mucociliary transport in a piglet model of cystic fibrosis. Science 2014;345:818-822.

51. Pezzulo AA, Tang XX, Hoegger MJ, Alaiwa MH, Ramachandran S, Moninger TO, Karp PH, Wohlford-Lenane CL, Haagsman HP, van Eijk $\mathrm{M}$, et al. Reduced airway surface $\mathrm{pH}$ impairs bacterial killing in the porcine cystic fibrosis lung. Nature 2012;487:109-113.

52. Keiser NW, Birket SE, Evans IA, Tyler SR, Crooke AK, Sun X, Zhou W, Nellis JR, Stroebele EK, Chu KK, et al. Defective innate immunity and hyper-inflammation in newborn CFTR-knockout ferret lungs. Am J Respir Cell Mol Biol (In press)

53. Zuelzer WW, Newton WA Jr. The pathogenesis of fibrocystic disease of the pancreas; a study of 36 cases with special reference to the pulmonary lesions. Pediatrics 1949;4:53-69. 